\title{
Objective evaluation of visibility in virtual chromoendoscopy for esophageal squamous carcinoma using a color difference formula
}

\author{
Masahito Inoue \\ Yoichi Miyake \\ Takeo Odaka \\ Toru Sato \\ Yoshiyuki Watanabe \\ Atsunori Sakama \\ Satoki Zenbutsu \\ Osamu Yokosuka \\ Chiba University \\ School of Medicine \\ Department of Endoscopic Diagnostics and Therapeutics \\ 1-8-1 Inohana, Chuo-ku \\ Chiba, Japan 260-8677
}

\begin{abstract}
Computed virtual chromoendoscopy with flexible spectral imaging color enhancement (FICE) is a new dyeless imaging technique that enhances mucosal and vascular patterns. However, a method for selecting a suitable wavelength for a particular condition has not been established. The aim of this study is to evaluate the color difference method for quality assessment of FICE images of the intrapapillary capillary loop in magnifying endoscopy for esophageal squamous cell carcinoma. The color difference between 60 microvessels and background mucosa observed using the magnifying endoscope was $8.31 \pm 2.84$ SD under white light and 12.26 $\pm 3.14(p$ $=0.0031), 11.70 \pm 4.49(p=0.0106)$, and 17.49 $55.40(p<0.0001)$ in FICE modes $A, B$, and $C$, respectively. The visibility scores for microvessels observed by medical students were $6.00 \pm 1.12$ points under white light and $11.1 \pm 2.25(p<0.0001), 8.65 \pm 2.06 \quad(p$ $=0.0001)$, and 12.55 $\pm 2.56(p<0.0001)$ in FICE modes A, B, and C, respectively. Furthermore, the measurement of color difference was correlated with the visibility score assigned by medical students (Pearson's correlation coefficient $=0.583, p<0.0001$ ) In conclusion, the color difference method corresponds to human vision and is an appropriate method for evaluation of endoscopic images. () 2010 Society of Photo-Optical Instrumentation Engineers. [DOI: 10.1117/1.3502666]
\end{abstract}

Keywords: endoscopy; image enhancement; visibility.

Paper 10054RR received Feb. 22, 2010; revised manuscript received Aug. 23, 2010 accepted for publication Aug. 31, 2010; published online Oct. 26, 2010.

\section{Introduction}

Esophageal squamous cell carcinoma accounts for $90 \%$ of esophageal cancer in Japan. The disease has a poor prognosis, and treatment of advanced carcinoma is especially invasive. ${ }^{1}$ Improvements in techniques such as endoscopic mucosal resection (EMR) and endoscopic submucosal dissection (ESD) have provided more radical treatment for early stage esophageal squamous cell carcinoma, ${ }^{2-4}$ but patients with invasion through the lamia propria into the muscularis mucosa or deeper may have lymph node metastases, ${ }^{1}$ and an accurate estimation of the tumor depth is required prior to treatment. Kumagai et al. invented a diagnostic method to determine tumor margins and depth by observing the intrapapillary capillary loop (IPCL) (microvessels at the surface of esophageal squamous carcinoma) using a magnifying endoscope. ${ }^{5}$ However, the color difference between the IPCL and background mucosa in esophageal squamous cell carcinoma is not always clear, and in this study, we evaluated the use of flexible spectral imaging color enhancement (FICE) and the CIE 1994 color difference method for quality assessment of endoscopic images of squamous cell carcinoma.

Address all correspondence to: Masahito Inoue, Chiba University, School of Medicine, Department of Endoscopic Diagnostics and Therapeutics, 1-8-1 Inohana, Chuo-ku, Chiba, Japan 260-8677. Tel: 81-43-226-2667; Fax: 81-43-2262667; E-mail: inouemasahito@hospital.chiba-u.jp

\section{Background}

Image enhancement plays a major role in current diagnosis by gastrointestinal endoscopy. FICE is an imaging enhancement system based on the technique of "spectrum estimation," which was developed by the Research Center for Frontier Medical Engineering, Chiba University. ${ }^{6}$ Use of this approach is becoming increasingly common. FICE involves irradiation of a subject with white light and taking of images using a charge-coupled device (CCD) camera in a regular endoscopic system. Composite FICE images are generated on a computer based on the imaging data obtained. The images taken by the CCD camera include data for the complete wavelength range of visible light; however, use of spectrum estimation allows viewing of an image taken under light at any specific wavelength. The FICE system can simultaneously process images under light at up to three wavelengths and can produce composite images under red, green, and blue light.

In narrow-band imaging (NBI), light of a specific wavelength is captured by a physical filter, rather than by a computer. $^{7}$ In the NBI system, irradiation is performed in a narrow band between 415 and $540 \mathrm{~nm}$, with white light that is passed through a filter positioned immediately in front of the light source. The light at $415 \mathrm{~nm}$ enhances visualization of

$1083-3668 / 2010 / 15(5) / 056019 / 6 / \$ 25.00$ ○ 2010 SPIE 
Inoue et al.: Objective evaluation of visibility in virtual chromoendoscopy...

Table 1 FICE wavelength and gain value settings (all wavelengths in $\mathrm{nm}$ ).

\begin{tabular}{|c|c|c|c|c|c|c|}
\hline & \multicolumn{2}{|c|}{ Red } & \multicolumn{2}{|c|}{ Green } & \multicolumn{2}{|c|}{ Blue } \\
\hline & Wavelength & Gain & Wavelength & Gain & Wavelength & Gain \\
\hline Mode A & 550 & 2 & 500 & 2 & 470 & 3 \\
\hline Mode B & 580 & 2 & 520 & 2 & 460 & 3 \\
\hline Mode C & 540 & 2 & 415 & 2 & 415 & 3 \\
\hline
\end{tabular}

blood vessels on the mucosal surface and that at $540 \mathrm{~nm}$ enhances blood vessels in deeper layers.

The major difference between FICE and NBI is the variability of the applicable wavelength-i.e., the wavelength in NBI is fixed and other wavelengths cannot be used. FICE is a promising method for use in various fields, including with regular and magnifying endoscopes, ${ }^{8,9}$ due to its ease of use and high flexibility regarding wavelength setting. An observer can freely set wavelengths in FICE, and the combinations of wavelengths are almost unlimited. However, the endoscopist has to select a suitable wavelength from many choices based on the situation, which makes the procedure more complex than NBI. Therefore, establishment of the wavelength mode corresponding to a particular use is likely to maximize the advantages of FICE and avoid unnecessary confusion in clinical practice.

Correct lesion diagnosis is the most important basis of evaluation of image enhancement in endoscopy. Results may be based on the diagnostic accuracy of hyperplasia, adenoma, or adenocarcinoma in colonic polyps ${ }^{10}$ or the demarcation line of a neoplastic lesion. ${ }^{9}$ However, the combinations of wavelengths in FICE are almost unlimited, and it is very difficult to compare the results using all these methods. Therefore, we chose to select wavelength settings with high efficacy in objective and automatic procedures and to evaluate the outcome based on human observation.

Image-engineering techniques use a color difference method for objective evaluation of the extent to which an observed item can be distinguished from the background. The CIE L*a*b system, a color system that is more similar to human color perception than the RGB color system, describes the color of an arbitrary point on a screen with lightness index $\left(L^{*}\right)$ and chromaticness indices $\left(a^{*}\right.$ and $\left.b^{*}\right)$, giving three elements $(L, a, b){ }^{11}$ The advantage of this color system is that it allows expression of the difference between two colors as a value, which is referred to as the "color difference." When two adjacent colors are measured and expressed as $\left(L_{1}, a_{1}\right.$, $\left.b_{1}\right)$ and $\left(L_{2}, a_{2}, b_{2}\right)$ in the CIE $\mathrm{L}^{*} \mathrm{a} * \mathrm{~b}$ system, the color difference $\left(\Delta E_{94}\right)$ is quantified by the following equation:

$$
\Delta E_{94}=\left[\Delta L^{2}+\left(\frac{\Delta C}{S_{C}}\right)^{2}+\left(\frac{\Delta H}{S_{H}}\right)^{2}\right]^{1 / 2},
$$

- $\Delta L=L_{1}-L_{2}$

- $\Delta C=C_{1}-C_{2}$

- $\Delta H=\sqrt{\Delta a^{2}+\Delta b^{2}+\Delta C^{2}}$

- $C_{1}=\sqrt{a_{1}^{2}+b_{1}^{2}}$
- $C_{2}=\sqrt{a_{1}^{2}+b_{2}^{2}}$

- $\Delta a=a_{1}-a_{2}$

- $\Delta b=b_{1}-b_{2}$

- $S_{C}=1+0.045 C_{1}$

- $S_{H}=1+0.015 C_{1}$

The color difference method is commonly used in the printing and publishing industries to evaluate color reproducibility and in other fields for evaluation of the visibility of advertisements, billboards, and signs. ${ }^{12}$

\section{Methods}

\subsection{Patients}

The subjects were 20 patients with early stage esophageal cancer who received endoscopic treatment in the Department of Endoscopic Diagnostics and Therapeutics, Chiba University Hospital (Chiba, Japan) from October 2006 to March 2008 and underwent magnification endoscopy combined with FICE prior to the operation. All examinations were performed by an endoscopist with 11 years of experience. Written informed consent for enrollment in the study was obtained from all patients in accordance with the Declaration of Helsinki.

\subsection{Endoscopy Equipment and Procedure}

A magnifying endoscope (EG-590ZW, Fujinon, Inc., Saitama, Japan) was used in the study, in combination with a light source (XL-4400, Fujinon, Inc.) and an image processor (VP4400, Fujinon, Inc.).

Of 10 factory default modes, 3 were used on the basis of a preliminary study (Table 1). Mode A has moderate brightness and vessel enhancement and is used for routine observation. Mode $\mathrm{B}$ gives priority to brightness rather than vessel enhancement and is used for observation of relatively dark and narrow sites, such as the large intestine. The wavelengths used in modes A and B were established by the manufacturer based on images taken under light at various wavelengths, with selection of the best wavelength for each mode. However, the criteria for each choice of wavelength require clarification. Mode C uses light at 525 and $495 \mathrm{~nm}$, at which spectral reflectance in the IPCL and normal esophageal mucosa is at a maximum. ${ }^{13}$ Therefore, this mode uses the optimal wavelength for observation of the IPCL.

All the patients were examined using a regular-power endoscope and diagnosed histopathologically with esophageal cancer prior to the study. An endoscopist with 11 years of experience inspected the lesions using an approximately 40- 


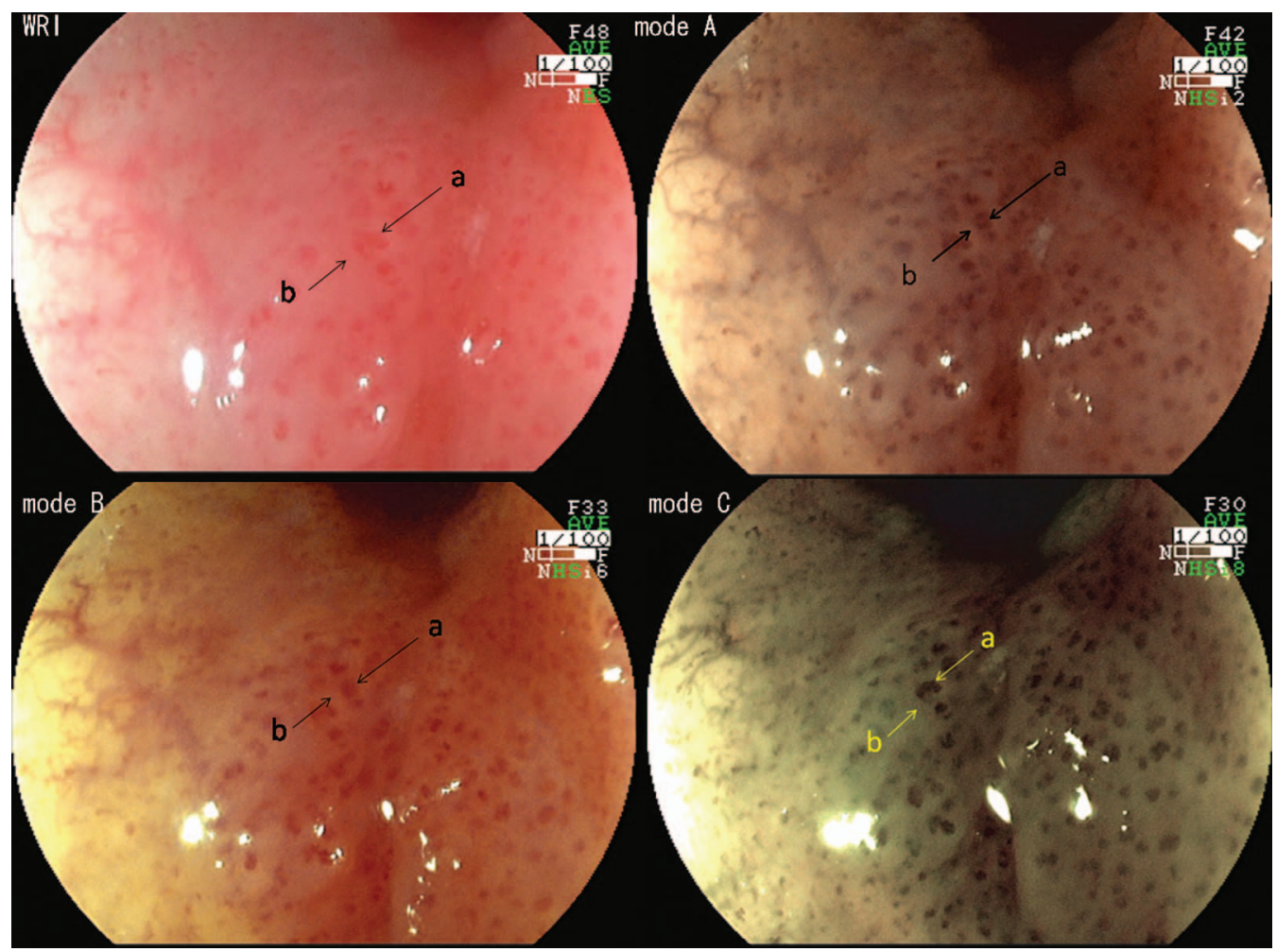

Fig. 1 Measurement of color difference using images taken by an approximately 50-power magnifying endoscope. White light-color of IPCL (a): $(L, a, b)=(53,53,33)$; color of background mucosa $(b):(L, a, b)=(54,47,26)$; color difference $\left(\Delta E_{94}\right)=2.94$. FICE mode A—Color of IPCL $(a)$ : $(L, a, b)=(25,26,21)$; color of background mucosa $(b):(L, a, b)=(40,18,19) ; \Delta E_{94}=17.12$. FICE mode B-Color of IPCL (a): $(L, a, b)$ $=(36,41,34)$; color of background mucosa $(b):(L, a, b)=(44,31,29) ; \Delta E_{94}=8.75$. FICE mode C—color of IPCL $(a):(L, a, b)=(17,6,9) ; \operatorname{color}$ of background mucosa (b): $(L, a, b)=(44,-3,20) ; \Delta E_{94}=29.21$.

to 60-power magnifying endoscope and took images in which the IPCL was observed under white light and in FICE modes $\mathrm{A}, \mathrm{B}$ and $\mathrm{C}$, respectively. All images of each lesion were taken in a single view to evaluate the same IPCL as far as possible. Three IPCLs in the images were chosen for evaluation. The color of each IPCL and the background mucosa were measured by the $\mathrm{L}^{*} \mathrm{a} * \mathrm{~b}$ method using Photoshop 6.0 (Adobe Systems, Inc.), and the color difference was calculated using the L*a*b equation (Figs. 1 and 2). To investigate matching of the evaluation based on the $\mathrm{L}^{*} \mathrm{a}^{*} \mathrm{~b}$ color difference with human vision, the visibility of the IPCL in the images was evaluated by five medical students from Chiba University who had no endoscopic experience. Endoscopic images for each subject were evaluated on the same liquid-crystal display as that used for measurement of color differences, in the following order: white light and FICE modes A, B, and C. The visibility of the IPCL under white light and in all FICE modes was judged as clear (4 points) when the shape and position of the IPCL were clearly visible; less clear (3 points) when the shape and position of the IPCL were visible, but not clearly; clouded (2 points) when the presence of the IPCL was apparent, but its position was difficult to see; and obscure (1 point) when the IPCL itself was difficult to see. The relationship between the mean color difference at three points in each image and evaluation scores given by five students was also determined. Last, the lesions were resected by EMR and diagnosed by pathological examination.

\subsection{Statistical Analysis}

Statmate version 3.16 was used for statistical analysis. A Bonferroni/Dunn test was performed to compare methods of color differentiation and macroscopic estimation with a $P$ value of less than 0.05 accepted as statistically significant. Pearson's rank correlation was performed to detect the correlation between color differentiation and macroscopic estimation.

\section{Results}

\subsection{Background Data}

The background data for the 20 patients are summarized in Table 2. The patients were all males and were age $71.9 \pm 7.06$ years (range 59 to 79 years old). All patients had early stage esophageal cancer with lesions of flat type in 5 cases and flat depressed type in 15 . The mean tumor diameter was $17.0 \pm 7.57 \mathrm{~mm}$ (range: 6 to $30 \mathrm{~mm}$ ). Regarding the tumor depth, tumors reached the epithelium (ep) in 10 lesions, the lamina propria (lpm) in 8 , and the muscularis mucosa $(\mathrm{mm})$ in 2 . The tumors comprised 6 well-differentiated, 13 moderately differentiated, and 1 poorly differentiated squamous carcinomas. No adenocarcinoma was found. All patients underwent endoscopic mucosal resection (EMR), and no additional treatment was required. 


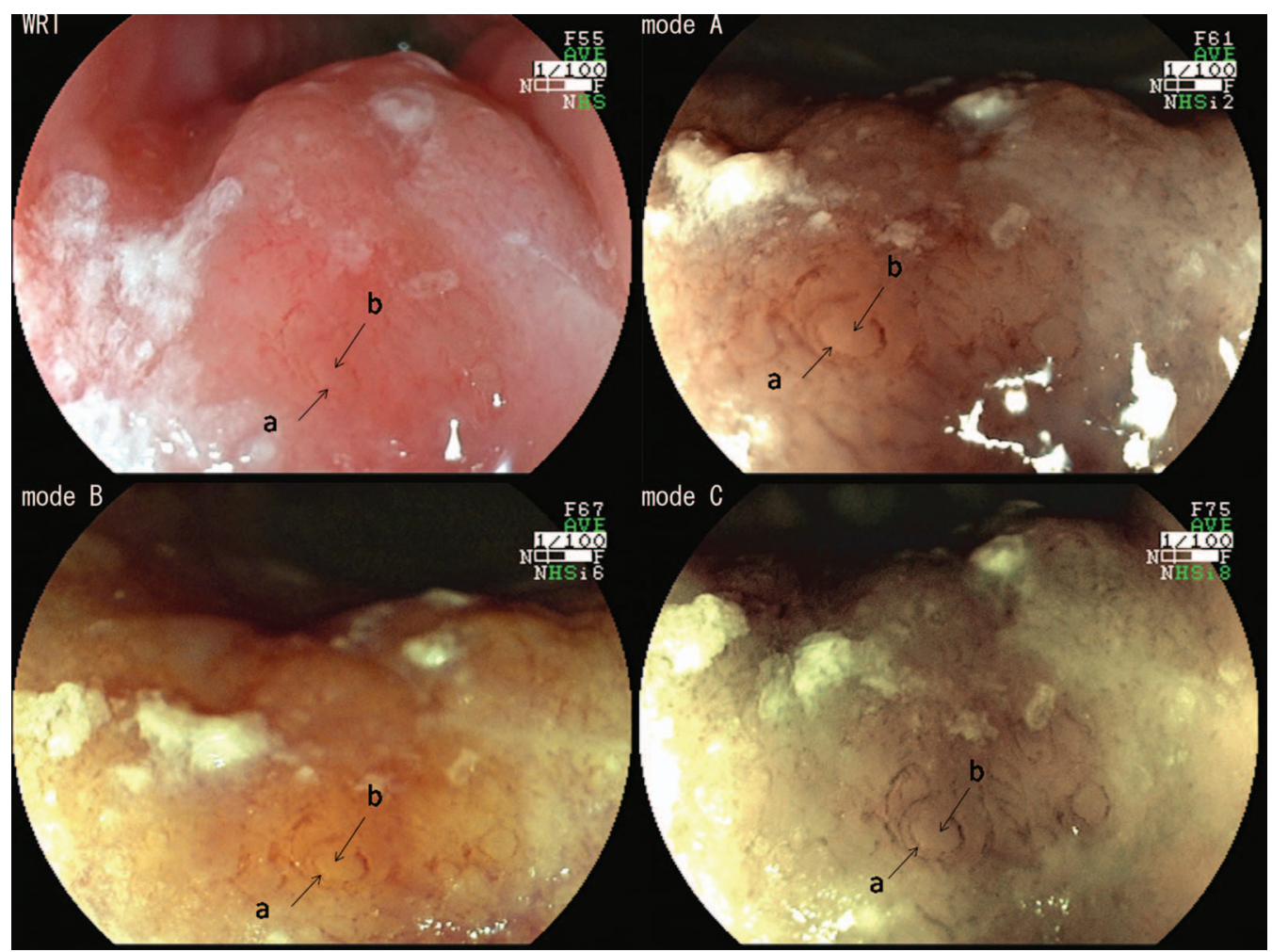

Fig. 2 Observation of flat elevated esophageal cancer by an approximately 50-power magnifying endoscope. White light-color of IPCL (a): $(L, a, b)=(50,41,29)$; color of background mucosa (b): $(L, a, b)=(65,29,20)$; color difference $\left(\Delta E_{94}\right)=15.69$. FICE mode A—Color of IPCL (a): $(L, a, b)=(28,30,28)$; color of background mucosa $(b):(L, a, b)=(53,23,28), \Delta E_{94}=25.25$. FICE mode B-color of IPCL $(a):(L, a, b)$ $=(41,36,37)$; color of background mucosa (b): $(L, a, b)=(59,23,45) ; \Delta E_{94}=19.94$. FICE mode C—Color of IPCL $(a):(L, a, b)=(11,13,13) ; \operatorname{color}$ of background mucosa (b): $(L, a, b)=(46,9,24) ; \Delta E_{94}=36.91$.

\subsection{Evaluation of the Color Difference between the IPCL and Background Mucosa}

Table 3 shows the individual data of the color difference and visibility score. The color difference between the IPCL and the background mucosa was measured at 3 points per image (i.e., 60 points total). This difference was $8.31 \pm 2.84$ under white light and $12.26 \pm 3.14(p=0.0031)$, and $11.70 \pm 4.49$ $(p=0.0106)$ and $17.49 \pm 5.40(p<0.0001)$ in FICE modes $\mathrm{A}, \mathrm{B}$, and $\mathrm{C}$, respectively. The color difference between the IPCL and the background mucosa was significantly greater in all FICE modes than under white light (Table 4).

\subsection{Evaluation of the Visibility of the IPCL by Medical Students}

The visibility scores for images were $6.00 \pm 1.12$ under white light and $11.1 \pm 2.25(p<0.0001), 8.65 \pm 2.06(p=0.0001)$, and $12.55 \pm 2.56(p<0.0001)$ in FICE modes A, B, and C, respectively. The scores for the IPCL in all FICE modes were significantly higher than the score under white light (Table 5). Furthermore, the measurement of color difference was correlated with the visibility score assigned by medical students (Pearson's correlation coefficient $=0.583, p<0.0001$ ).

The preceding results show (1) that evaluation of FICE images by measurement of the color difference corresponded to evaluation by the human eye and (2) that FICE modes A and C significantly enhanced the "visibility" of the IPCL in
Table 2 Characteristics of 20 patients with early esophageal carcinoma.

\begin{tabular}{ll}
\hline Variable & Value \\
\hline Mean age (years) & $71.9(59$ to 79$)$ \\
\hline Sex & 20 \\
Mistopathology & \\
Squamous cell carcinoma & \\
Well-differentiated type & 6 \\
Moderately differentiated type & 13 \\
Poorly differentiated type & 1 \\
\hline Mean size of lesions (mm) & 17.0 (6 to 30) \\
\hline Macroscopic type & 15 \\
Flat type & 5 \\
Slightly depressed type &
\end{tabular}


Inoue et al.: Objective evaluation of visibility in virtual chromoendoscopy...

Table 3 Individual data of the color difference and visibility score.

\begin{tabular}{|c|c|c|c|c|c|c|c|c|}
\hline \multirow[b]{2}{*}{ Patient } & \multicolumn{4}{|c|}{ Average of color difference } & \multicolumn{4}{|c|}{ Visibility score } \\
\hline & WRI & Mode A & Mode B & Mode C & WRI & Mode A & Mode B & Mode C \\
\hline 1 & 10.15 & 16.61 & 12.31 & 25.95 & 7 & 12 & 7 & 8 \\
\hline 2 & 6.13 & 16.03 & 12.23 & 15.96 & 6 & 11 & 9 & 10 \\
\hline 3 & 14.25 & 12.58 & 16.2 & 19.59 & 7 & 8 & 9 & 12 \\
\hline 4 & 5.37 & 9.39 & 8.33 & 19.89 & 6 & 9 & 5 & 12 \\
\hline 5 & 6.37 & 17.17 & 12.55 & 15.89 & 5 & 12 & 5 & 12 \\
\hline 6 & 9.84 & 10.73 & 12.09 & 13.55 & 5 & 12 & 8 & 12 \\
\hline 7 & 9.37 & 11 & 12.49 & 21.17 & 5 & 11 & 8 & 14 \\
\hline 8 & 11.49 & 15.74 & 10.29 & 14.62 & 5 & 11 & 8 & 14 \\
\hline 9 & 12.76 & 13.11 & 26.57 & 26.71 & 5 & 12 & 9 & 12 \\
\hline 10 & 9.8 & 11.31 & 10.16 & 23.01 & 5 & 11 & 10 & 12 \\
\hline 11 & 8.81 & 11.48 & 15.29 & 16.78 & 5 & 13 & 9 & 11 \\
\hline 12 & 6.15 & 14.26 & 11.59 & 19.22 & 8 & 15 & 14 & 17 \\
\hline 13 & 7.45 & 9.45 & 9.21 & 13.45 & 7 & 12 & 9 & 14 \\
\hline 14 & 4.23 & 9.21 & 5.66 & 9.23 & 6 & 11 & 7 & 13 \\
\hline 15 & 8.31 & 13.26 & 11.36 & 23.11 & 8 & 15 & 12 & 19 \\
\hline 16 & 11.23 & 12.34 & 13.79 & 21.2 & 6 & 9 & 9 & 13 \\
\hline 17 & 5.91 & 11.24 & 11.34 & 13.24 & 5 & 6 & 6 & 10 \\
\hline 18 & 6.98 & 7.25 & 6.76 & 8.23 & 5 & 6 & 6 & 10 \\
\hline 19 & 8.12 & 14.34 & 8.12 & 18.34 & 8 & 13 & 10 & 15 \\
\hline 20 & 3.45 & 6.81 & 5.65 & 9.59 & 5 & 8 & 6 & 9 \\
\hline
\end{tabular}

esophageal squamous cell carcinoma lesions in magnification endoscopy compared to white light.

\section{Discussion}

Images visualized by magnification endoscopy differ substantially between esophageal adenocarcinoma and squamous cell carcinoma. Adenocarcinoma, which accounts for $50 \%$ or more of cases in Europe and the United States, ${ }^{14}$ can be identified by pit pattern classification. ${ }^{15,16}$ The prevalence of Barrett's esophagus is lower in Japan than in Europe and the United States, and squamous cell carcinoma accounts for $90 \%$ of esophageal cancer. ${ }^{1}$ The intrapapillary capillary loop (IPCL) is commonly observed in squamous cell carcinoma derived from the squamous epithelium with little or no glandular structure. Inoue ${ }^{17,18}$ and Arima et al. ${ }^{19}$ showed that carcinoma gradually destroys normal microvessels with subsequent development of abnormal neovessels and avascular areas, leading to establishment of a method for diagnosis of the tumor margin and depth based on morphological classification. However, the color of the IPCL is similar to that of the background mucosa, which often prevents recognition. Use of an image enhancement system such as narrow-band imaging (NBI) improves the visibility of carcinoma, ${ }^{7}$ since NBI en-

Table 4 Evaluation of the color difference between the IPCL and the background mucosa.

\begin{tabular}{lll}
\hline & $\Delta E$ & $P$ value \\
\hline WRI & $8.31 \pm 2.84 \mathrm{SD}$ & \\
FICE mode A & $12.26 \pm 3.14 \mathrm{SD}$ & $=0.0031$ \\
FICE mode B & $11.70 \pm 4.49 \mathrm{SD}$ & $=0.0106$ \\
FICE mode C & $17.49 \pm 5.40 \mathrm{SD}$ & $<0.0001$ \\
\hline
\end{tabular}


Inoue et al.: Objective evaluation of visibility in virtual chromoendoscopy...

Table 5 Evaluation of the visibility of the IPCL by medical students.

\begin{tabular}{lll}
\hline & Score & $P$ value \\
\hline WRI & $4.46 \pm 1.75 \mathrm{SD}$ & \\
FICE mode A & $10.0 \pm 1.34 \mathrm{SD}$ & $<0.0001$ \\
FICE mode B & $6.29 \pm 1.25 \mathrm{SD}$ & 0.0006 \\
FICE mode C & $10.64 \pm 1.43 \mathrm{SD}$ & $<0.0001$ \\
\hline
\end{tabular}

hances the IPCL and microstructures on the mucosal surface by narrowing the band of white light using a filter attached to the light source. ${ }^{20}$

The results of the current study showed a significant improvement in detection of the color difference in observation by a magnifying endoscope combined with FICE compared with that under white light, regardless of the FICE mode. However, the performance in mode $\mathrm{C}$ was the best, followed by modes $\mathrm{A}$ and $\mathrm{B}$, respectively. The results of observation by medical students showed a correlation with the FICE data, which shows that the color difference method corresponds to human vision and is an appropriate method for evaluation of endoscopic images. Mode $\mathrm{C}$, the best setting, uses two wavelengths at which the spectral reflectance of the IPCL and background mucosa differed substantially from each other, as determined in the development process. Specifically, the setting based on histological and image-engineering rationales was recognized to be appropriate in evaluation of visibility. We cannot conclude that mode $\mathrm{C}$ is the best wavelength setting for esophageal observation using a magnifying endoscope among all possible settings, and its utility requires confirmation in further comparisons with other settings. Conversely, a setting superior to mode $\mathrm{C}$ may be found. In this study, we focused on observation of the IPCL using an esophageal magnifying endoscope. However, the color difference method can be applied to the lesion margin in regular endoscopy and for evaluation of pit visibility in magnification endoscopy, and we plan to find the most suitable FICE wavelength setting for each use.

\section{Acknowledgments}

I am deeply grateful to the late Teruo Kouzu, who endeavored to develop FICE. His comments and suggestions were of inestimable value for my study.

\section{References}

1. K. Takubo, J. Aida, M. Sawabe, M. Kurosumi, M. Arima, M Fujishiro, and T. Arai, "Early squamous cell carcinoma of the oesophagus, the Japanese viewpoint," Histopathology 51, 733-742 (2007).
2. M. Ciocirlan, M. G. Lapalus, V. Hervieu, J. C. Souquet, B. Napoleon, J. Y. Scoazec, C. Lefort, J. C. Saurin, and T. ponchon, "Endoscopic mucosal resection for squamous premalignant and early malignant lesions of the esophagus," Endoscopy 39, 24-29 (2007).

3. H. Makuuchi, "Esophageal endoscopic mucosal resection (EEMR) tube," Surg. Laparosc Endosc 6, 160-161 (1996).

4. T. Oyama, A. Tomori, K. Hotta, S. Morita, K. Kominato, M. Tanaka, and Y. Miyata, "Endoscopic submucosal dissection of early esophageal cancer," Clin. Gastroenterol. Hepatol. 3, S67-70 (2005).

5. Y. Kumagai, H. Inoue, K. Nagai, T. Kawano, and T. Iwai, "Magnifying endoscopy, stereoscopic microscopy, and the microvascular architecture of superficial esophageal carcinoma," Endoscopy 34, 369-375 (2002).

6. Y. Miyake, T. Sekiya, and T. Hara, "A new spectrophotometer for measuring the spectral reflectance of gastric mucous membrane," $J$. Photogr. Sci. 37, 134-138 (1989).

7. K. Goda, H. Tajiri, M. Ikegami, Y. Yoshida, N. Yoshimura, M. Kato, K. Sumiyama, H. Imazu, K. Matsuda, M. Kaise, T. Kato, and S. Omar, "Magnifying endoscopy with narrow band imaging for predicting the invasion depth of superficial esophageal squamous cell carcinoma," Dis. Esophagus 13, 453-460 (2009).

8. J. Pohl, A. May, T. Rabenstein, O. Pech, and C. Ell, "Computed virtual chromoendoscopy, a new tool for enhancing tissue surface structures," Endoscopy 39, 80-83 (2007).

9. H. Osawa, M. Yoshizawa, H. Yamamoto, H. Kita, K. Satoh, H Ohnishi, H. Nakano, M. Wada, M. Arashiro, M. Tsukui, K. Ido, and K. Sugano, "Optimal band imaging system can facilitate detection of changes in depressed-type early gastric cancer," Gastrointest. Endosc. 67, 226-234 (2008).

10. J. Pohl, E. Lotterer, C. Balzer, et al., "Computed virtual chromoendoscopy versus standard colonoscopy with targeted indigocarmine chromoscopy, a randomised multicentre trial," Gut 58, 73-78 (2009).

11. CIE, "Recommendations on uniform color spaces, color difference equations, psycometric color terms," CIE Publication 15 (1971).

12. CIE, "Industrial color-difference evaluation," CIE Publication 116 (1995).

13. Y. Miyake, T. Kouzu, S. Takeuchi, et al., "Development of new electronic endoscopes using the spectral images of an internal organ," in Proc. 13th Color Imaging Conf. (CIC13), Scottsdale, AZ, pp. 261263 (Nov. 2005).

14. S. S. Devesa, W. J. Blot, and J. F. Fraumeni Jr., "Changing patterns in the incidence of esophageal and gastric carcinoma in the United States," Cancer 83, 2049-2053 (1998).

15. W. L. Curvers, C. J. Bohmer, R. C. Mallant-Hent, A. H. Naber, C. I. Ponsioen, K. Ragunath, R. Singh, and M. B. Wallace, "Mucosal morphology in Barrett's esophagus, interobserver agreement and role of narrow band imaging," Endoscopy 40, 799-805 (2008).

16. P. Sharma, A. P. Weston, M. Topalovski, R. Cherian, A. Bhattacharyya, and R. E. Sampliner, "Magnification chromoendoscopy for the detection of intestinal metaplasia and dysplasia in Barrett's oesophagus," Gut 52, 24-27 (2003).

17. H. Inoue, "Magnification endoscopy in the esophagus and stomach," Dig. Endosc. 13, 40-42 (2001).

18. H. Inoue, T. Honda, and T. Yoshida, "Ultra-high magnification endoscopy of the normal esophageal mucosa," Dig. Endosc. 8, 16-18 (1996).

19. M. Arima, M. Tada, and H. Arima, "Evaluation of microvascular patterns of superficial esophageal cancers by magnifying endoscopy," Esophagus 4, 191-197 (2005).

20. K. Gono, T. Obi, M. Yamaguchi, N. Ohyama, H. Machida, Y. Sano, S. Yoshida, Y. Hamamoto, and T. Endo, "Appearance of enhanced tissue features in narrow-band endoscopic imaging," J. Biomed. Opt. 9, 568-577 (2004) 